\title{
ERRATUM
}

\section{Erratum to: SWAAT Study: Extracorporeal Shock Wave Therapy and Arginine Supplementation and Other Nutraceuticals for Insertional Achilles Tendinopathy}

Angela Notarnicola $\cdot$ Vito Pesce $\cdot$ Giovanni Vicenti · Silvio Tafuri · Maria Forcignanò $\cdot$ Biagio Moretti

(C) Springer Healthcare 2012

Erratum to: Notarnicola A, Pesce V, Vicente G, et al. SWAAT Study: Extracorporeal Shock Wave Therapy and Arginine Supplementation and Other Nutraceuticals for Insertional Achilles Tendinopathy. Adv Ther. 2012;29(9):799-814.

The authors of the above-mentioned paper would like to make the following adjustments to their article:

In the Abstract, the first line of the Conclusion should read:

On the basis of the results obtained in this study, the authors conclude that in the treatment of insertional Achilles tendinopathy, ESWT induces a hemodynamic re-equilibrium with an amelioration in tendon trophism.

In the Conclusion section of the article, the first line of the final paragraph should read:

In conclusion, although a small sample of patients was involved and only small differences were detected between the two groups in the present study, the findings suggest that dietary supplement plus ESWT can induce a better clinical and functional outcome and a reduction in tendon neovascularization in patients with Achilles tendinopathy.

The authors thank Springer Healthcare for publishing the corrections. 